Check for updates

Cite this: RSC Adv., 2017, 7, 30784

Received 10th April 2017

Accepted 8th June 2017

DOI: $10.1039 / c 7 r a 04074 a$

rsc.li/rsc-advances

\section{Detection of vasodilator drugs through microwave spectroscopy of Au NP colorimetric probes using a microwave metallic photonic crystal-inspired resonant probe $\dagger$}

\begin{abstract}
Rahul Yadav, (D) a Piyush N. Patel (D) and V. N. Lad (D) *b
Here, we report a low-cost, time-effective, easy-to-use, and novel analytical technique incorporating microwave spectroscopy of unmodified gold nanoparticles for the successful detection of two vasodilator drugs - amlodipine besylate and minoxidil. The sensor probe consists of a microwave metallic photonic crystal, which is loaded into the ground plane of a $\sim \lambda / 4$ cylindrical line source. The sensing scheme is based on inducing aggregation in the gold nanoparticles upon addition of the analyte drug, and then performing microwave spectroscopy for different concentrations of the two drugs. The proposed method was validated in the concentration ranges of 100-0.05 $\mu \mathrm{M}$ and 20-0.625 mM for amlodipine besylate and minoxidil drugs, respectively, and a good linearity was obtained for the calibrated data. The scope of the reported user-friendly technique can be further enhanced by improvement of the limit of detection of specific drugs by favourable functionalization and/or surface modification of suitable nanoparticle probes.
\end{abstract}

\section{Introduction}

Vasodilator drugs are effective in preventing numerous health disorders like high blood pressure, heart failure, and angina. ${ }^{1}$ Most of the vasodilator drugs are balanced with arterial dilators and venous dilators. They are used to dilate arteries and veins simultaneously. Therefore, they prove to be beneficial as an enhanced therapeutic treatment. ${ }^{2}$ However, consumption of the vasodilator may interfere with other dietary elements and may cause nausea, headaches, heart palpitations, etc. amlodipine besylate is an L-type calcium channel blocker which is used for the treatment of angina and hypertension. It also has antihypertensive and antianginal effects. ${ }^{3}$ Minoxidil is used to control blood pressure by relaxing and dilating the blood vessels, and it is always used in combination with beta-blockers and diuretics in order to prevent its side effects. ${ }^{4}$ Several analytical techniques such as High Pressure Liquid Chromatography (HPLC), ${ }^{5,6}$ Liquid Chromatography-Mass Spectrometry (LC-MS), ${ }^{7,8}$ fluorescence, ${ }^{9,10}$ electrochemical, ${ }^{11,12}$ and UV-visible spectroscopy ${ }^{13,14}$ are reported for the analysis of two vasodilator drugs (amlodipine besylate and minoxidil) in pure pharmaceutical formulations and tablets. Unfortunately, these methods require more time for analysis,

${ }^{a}$ Sensor Research Laboratory, Electronics Engineering Department, Sardar Vallabhbhai National Institute of Technology, Surat, 395007, India. E-mail: ryrahulyadav01@ gmail.com; piyushsvnit@gmail.com

${ }^{b}$ Chemical Engineering Department, Sardar Vallabhbhai National Institute of Technology, Surat, 395007, India. E-mail: vnl@ched.svnit.ac.in

$\dagger$ Electronic supplementary information (ESI) available. See DOI: 10.1039/c7ra04074a expensive instrumentation, and moreover, their applicability is limited only to the laboratory. Therefore, a simple analytical technique is essential for the detection and quantification of analyte drugs in different matrices.

The gold nanoparticles (Au NPs) as colorimetric probes are actively involved in research for the development of effective detection and screening approach of biomolecules, inorganic elements and drugs. ${ }^{15}$ The particle distance-dependent optical property of the Au NPs undergoes red-shift in the wavelength of surface plasmon resonance (SPR) after the induced aggregation and exhibits a very high extinction coefficient. ${ }^{16}$ This unique tunable optical property sensitively allows Au NPs for visual sensing of the analyte through color change with great sensitivity. It is found that small sized $(\sim 5-13 \mathrm{~nm})$ water dispersible Au NPs play an important role in the colorimetric assay. In this connection, unmodified Au NPs and functionalized Au NPs have been utilized as probes for the colorimetric detection of target drug molecules by creating affinity and inducing aggregation. Rawat et al. showed simultaneous detection of four different drug formulations (venlafaxine, imipramine, amlodipine, and alfuzosin) by using bare gold nanoparticles. It is achieved through strong interaction between Au NPs surface and four drugs at $\mathrm{pH} 4$ ammonium acetate buffer media. ${ }^{17}$ Teepoo et al. applied colorimetric assay using unmodified Au NPs for the determination of drug ramoplanin in biofluid up to $0.01 \mathrm{ppm} .{ }^{18}$ Yarbakht and coworker reported the visual detection of methamphetamine using aptamer coated gold nanoparticles. ${ }^{19}$ Xie et al. developed DNA interstrand cross-linked Au NPs for colorimetric detection of the anticancer drug. ${ }^{20}$ Selvaraj et al. showed biological assay of 
antileukemic drug (6-mercaptopurine) using gold nanoparticles. ${ }^{21}$ These approaches allow simple analysis for detecting the presence of analyte drug; however, as the analyte concentration approaches the limit of detection (LOD), the UV-visible spectrometry fail to deliver highly distinct absorbance spectra. Thus, calibration is one of the issues which restrict the development of a portable colorimetric-based spectrometer as a prototype sensor device. This encouraged us to introduce an ingenious sensor and spectroscopic study which possesses a potential scope in the development of the novel portable device to facilitate effective detection of the concerned species.

Devices based on microwave theory and techniques have also gained significant attention for bio-sensing. In the recent years, resonator-based radiofrequency (RF) biosensing platforms have been developed for sensing over a broad range of microwave spectrum. Grenier et al. presented a broadband microwave sensor integrated with microfluidic technology dedicated for the purpose of bioengineering the human cells. ${ }^{22}$ Compact coplanar waveguide transmission line based label-free RF biosensors are reported for the characterization of cancer cell, ${ }^{23}$ glucose ${ }^{24}$ and many other analytes. More interestingly, artificial materials such as metamaterials have emerged with excellent sensing properties at nanostructured scale. ${ }^{25}$ Cao et al. developed $\sim 45 \mu \mathrm{m}$ width U-shaped periodic structure of Split Ring Resonators (SRRs) with the thiolated surface to detect the presence of $\mathrm{RGG}_{9}$ biomarker. ${ }^{26}$ Park et al. reported electric LCtype terahertz metamaterials for the characterization of fungi bacteria. ${ }^{27}$ In particular, metamaterials operating at terahertz frequencies and the optical regime have a relatively higher sensitivity. However, expensive fabrication, complex measurement setup, and sample preparation with remarkably sophisticated functionalization is required. Whereas, X-band (8-12 $\mathrm{GHz}$ ) metamaterial sensor demonstrated by Lee and co-authors for specific binding between prostate-specific antigen (PSA) and antibody showed LOD up to $1 \mathrm{ng} \mathrm{mL}^{-1} \cdot{ }^{28}$ Since the majority of the investigated microwave sensor devices are planar, in situ analysis cannot be performed into various biological and chemical assays. Microwave reflection probes are one of the suitable candidates for the in situ microwave spectroscopy. But, so far, only the non-resonant open-ended coaxial probes have been studied for the broadband microwave dielectric spectroscopy of liquids, ${ }^{29}$ biological tissues, ${ }^{30}$ and RF laminates. ${ }^{31}$ Large sample volume is required in some of the exceptionally designed yet bulky resonant probes. ${ }^{32,33}$

In this work, a low-profile coaxial probe is presented which acquires resonance from the microwave metallic photonic crystal loaded in the probe ground plane. The sensing mechanism is based on microwave spectroscopy of the gold nanoparticles colorimetric assay, wherein, the probe tip is inserted into the prepared sample and shift is calibrated in the resonant frequency. The aggregation in Au NPs by the two drugs (amlodipine besylate, and minoxidil) which led to the red-shift in the wavelength of SPR was confirmed using a UV-visible spectrophotometer, Dynamic Light Scattering (DLS), and FT-IR measurements. The proposed technique possesses the advantage of simple measurement setup, significantly identifiable resonance peaks, minimal requirement of sample volume, and easy calibration.

\section{Sensor development}

\subsection{Design}

The design of metallic photonic crystal (MPC) loaded microwave probe consists of a four surrounded rectangular metal rods, each with the width of $w_{1}=2 \mathrm{~mm}$, height $L_{1}=7.5 \mathrm{~mm}$, and vertically suspended on the ground plane of a panel type SMA female connector of ground plane length $L=12.5 \mathrm{~mm}$. The cylindrical pin of diameter $d=1.3 \mathrm{~mm}$ and height $l_{1}=16.3 \mathrm{~mm}$ act as a $\sim \lambda / 4$ line source carrying current, where, $\lambda$ is the operating wavelength. Fig. 1a shows the schematic of the proposed sensor probe, whose transverse magnetic (TM) bandgap regions were numerically calculated using Eigenmode solver of the Computer Simulation Tool, Microwave Studio (CST-MWS) v.2016. The finite line source having a current density $(J)$ traps the evanescent waves within the MPC, and energy is allowed to propagate only above the MPC structure. Thus, the entire field components no longer remain invariable in the $z$-direction for TM boundary and it leads to the existence of bandgap. It is well-known that supper-cell structure is commonly applied by defining the periodic boundary to the

(a)

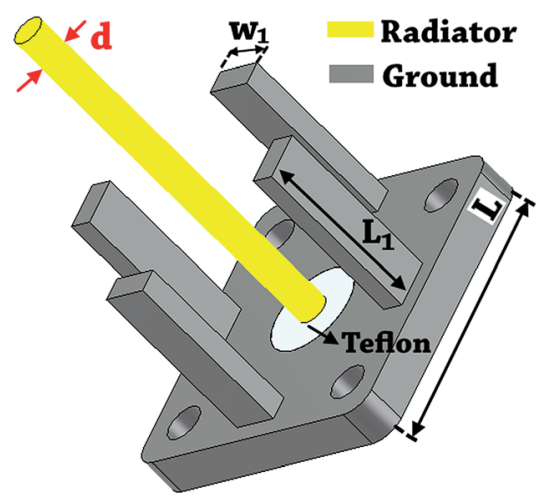

(b)

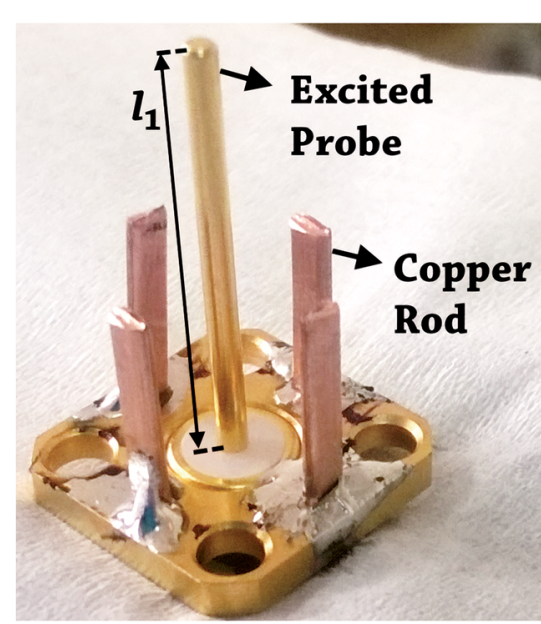

Fig. 1 (a) Schematic of the proposed resonant probe. (b) Fabricated structure. 


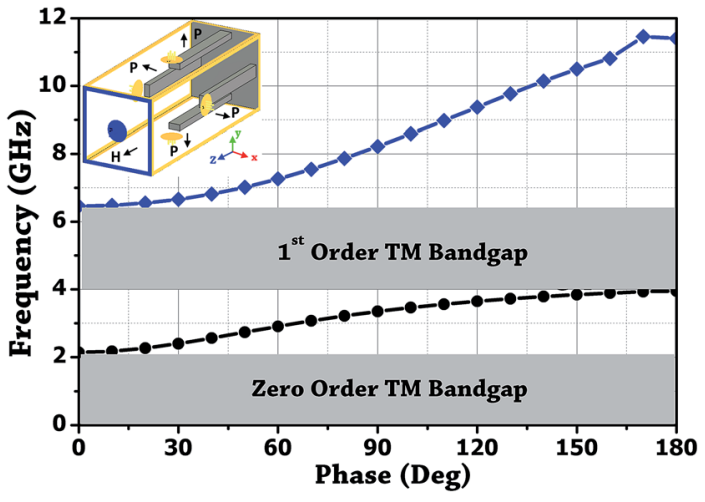

Fig. 2 Dispersion diagram of microwave metallic photonic crystal in square lattice.

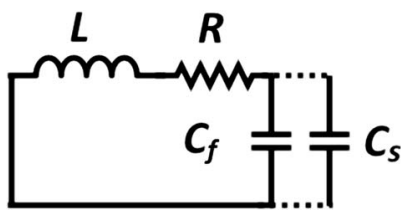

Fig. 3 Equivalent lumped circuit model.

unit-cell structure for the computation of microwave photonic crystal. ${ }^{34}$ However, in the proposed geometry, the lattice points are in the middle of the square lattice plane, and therefore, computation burden can be minimized by calculating phase variation in $x$ or $y$-direction only, and the irreducible Brillouin zone boundary need not be evaluated completely. ${ }^{35}$ Fig. 2 shows the simulated bandgaps for TM mode, where, zero order global TM bandgap is up to $2 \mathrm{GHz}$, and $1^{\text {st }}$ order TM bandgap exists between $4 \mathrm{GHz}$ to $6.2 \mathrm{GHz}$. Based on these results, the probe resonance is likely to exist in the $1^{\text {st }}$ order TM bandgap region. As shown in Fig. 3, the sensing operation can be understood from the equivalent probe circuit. The lumped model of the excited coaxial line has a finite inductance $(L)$ and resistance $(R)$, whereas, the $C_{\mathrm{f}}$ is the fringing field capacitance between the probe and MPC rods.

In this case, the resonant frequency can be obtained using eqn (1). When the probe tip interacts with the liquid sample, the value of $C_{\mathrm{f}}$ is influenced and acts as an additional capacitance $C_{\mathrm{s}}$ in parallel with the sample as shown in eqn (2). This parallel capacitance, therefore, shifts down the resonant frequency $\left(f_{\mathrm{r}}\right)$.

$$
\begin{gathered}
f_{\mathrm{r}}=\frac{1}{2 \pi \sqrt{L C_{\mathrm{f}}}} \\
f_{\mathrm{r}}=\frac{1}{2 \pi \sqrt{L\left(C_{\mathrm{f}}+C_{\mathrm{s}}\right)}}
\end{gathered}
$$

\subsection{Device fabrication and testing}

The probe was realized by utilizing a female sub-miniature version-A (SMA) panel type connector with elongated feed pin and soldering four rectangular copper rods over the ground body of the connected which surrounds the feed pin. Fig. 1b

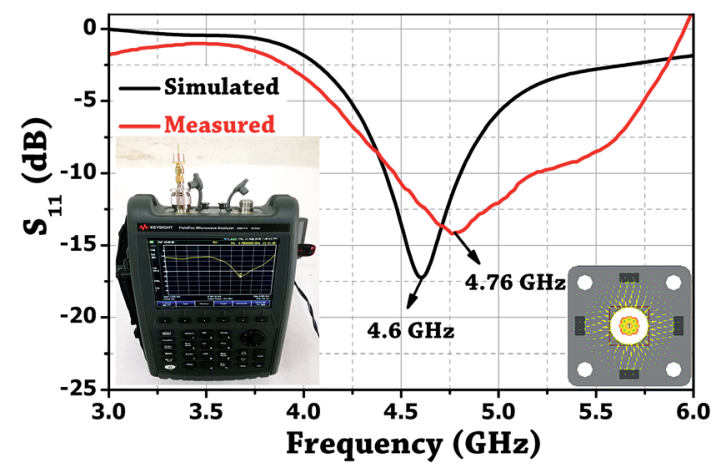

Fig. 4 Plot of the reflection coefficient for the MPC-inspired resonant probe.

shows the photo of the fabricated probe. A microwave signal excitation was given to the probe, and its reflection scattering spectra $\left(S_{11}\right)$ was simulated and later measured using Vector Network Analyzer (VNA) to validate the proof of concept. It is evident from Fig. 4 that the simulated fundamental resonance of the probe at $4.6 \mathrm{GHz}$ is found to be in the $1^{\text {st }} \mathrm{TM}$ bandgap and possess $\mathrm{TM}_{110}$ mode. The measured frequency response at 4.76 $\mathrm{GHz}$ is in close agreement with the simulation result and confirms its proper functioning.

\section{Experiment}

\subsection{Chemicals and materials}

Gold chloride $\left(\mathrm{HAuCl}_{4} \cdot x \mathrm{H}_{2} \mathrm{O}\right)$ and sodium citrate tribasic dihydrate $\left(\mathrm{C}_{6} \mathrm{H}_{5} \mathrm{Na}_{3} \mathrm{O}_{7} \cdot 2 \mathrm{H}_{2} \mathrm{O}\right)$ were purchased from SRL Pvt. Ltd, India. TRIS (hydroxymethyl) aminomethane, phosphate buffer saline (PBS) and ammonium acetate were purchased from SigmaAldrich, USA. Amlodipine besylate and minoxidil were obtained from TCI Chemicals, Japan. The milli-Q-pure water of $18.2 \mathrm{M} \Omega$ was used in the experimentation. All the reagents were of analytical grade and used without further purification.

\subsection{Instrumentation}

The probe resonant frequency was measured using Agilent vector network analyzer (N9917A, $18 \mathrm{GHz}$ ). The UV-visible absorbance spectrum was measured with Ocean Optics, Maya Pro 2000 spectrophotometer. DLS analysis was performed using Zetasizer Nano ZS90, Malvern Instruments, and FTIR spectroscopy was recorded with the help of ATR-8000A, Shimadzu.

\subsection{Synthesis and characterization of gold nanoparticles}

The $\mathrm{Au}$ NPs were prepared by the reduction of $\mathrm{Au}^{3+}$ using Turkevich method. ${ }^{36}$ In brief, $20 \mathrm{~mL}$ of $1 \mathrm{mM} \mathrm{HAuCl}_{4}$ was refluxed in a $50 \mathrm{~mL}$ conical flask for $15 \mathrm{~min}$ under constant stirring. To this, $38.8 \mathrm{mM}$ of citrate was rapidly injected, and the mixture was further stirred for $40 \mathrm{~min}$ at $510 \mathrm{rpm}$. Maintaining the reaction temperature at $\pm 85{ }^{\circ} \mathrm{C}$ yielded change in the colour of the solution from pale yellow to cherry red and confirms the formation of Au NPs. The peak absorbance of the synthesized Au NPs was measured at $519 \mathrm{~nm}$, and a resonance at $3.4 \mathrm{GHz}$ was obtained by dipping the probe tip in the colloidal solution 
of gold as shown in Fig. 5. It is important to understand that it is the solution permittivity which causes the resonant frequency to shift towards the lower side in the spectrum as, $f \propto \frac{1}{\sqrt{\varepsilon}}$, where $f$ is the frequency and $\varepsilon$ is the solution permittivity.

\subsection{Detection of drugs}

Initially, colorimetric detection of two vasodilator drugs (amlodipine besylate and minoxidil) was independently carried
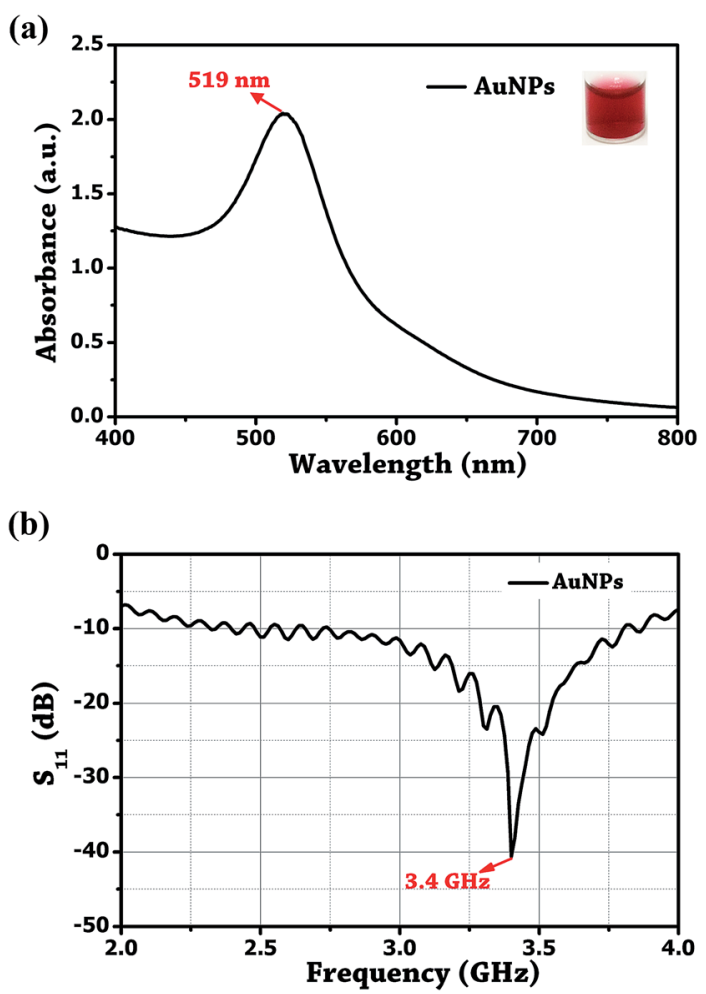

Fig. 5 (a) UV-visible absorbance plot. (b) Probe reflection coefficient $\left(S_{11}\right)$ of the unmodified Au NPs. out using unmodified Au NPs as sensing probe. The details of the drugs are illustrated in ESI Table $1 . \dagger$ Briefly, $100 \mu \mathrm{L}$ of different concentrations of amlodipine and minoxidil was prepared in deionized water and separately added into $500 \mu \mathrm{L}$ of unmodified $\mathrm{Au}$ NPs. The resulting color change in the solution after $5 \mathrm{~min}$ of incubation at room temperature was analyzed with UV-visible spectroscopy, DLS, and FTIR measurement to study the aggregation mechanism of Au NPs with the drugs. Immediately, after the induced aggregation, the microwave sensor probe tip was dipped into the solution, and a corresponding shift in the resonant frequency was recorded. Thus, the detection of two vasodilator drugs is based microwave spectroscopy of the aggregated Au NPs, where, the aggregation phenomenon of Au NPs amplifies the signal response of the probe unlike performing microwave spectroscopy of samples in purified water. This technique is useful when the color change in the solution cannot be distinguished with naked eyes and the UV-visible spectroscopic results show ambiguity in the region of peak absorption (ESI Fig. 2†). Next, the frequency responses were calibrated to find the LOD.

\section{Results and discussion}

\subsection{Sensing mechanism and measurement technique}

The solution $\mathrm{pH}$ is one the key parameter which enables selective probing of the gold nanoparticles with the functional groups of target drug molecules. Since the dissociation constant $\left(\mathrm{p} K_{\mathrm{a}}\right)$ value of amlodipine besylate is $8.6,{ }^{37}$ and that of minoxidil is $4.61,{ }^{38} \mathrm{pH} 4$ buffer media was selected to facilitate a positive charge on the drug molecules. At this $\mathrm{pH}$, the surface of the citrate-stabilized Au NPs acquires negative change, because of first $\mathrm{p} K_{\mathrm{a}}$ value of citrate being $<4$, and electrostatically interacts with the molecules of the positively charged drug. Furthermore, the active functional group $\left(-\mathrm{NH}_{2}\right)$, electron rich $\mathrm{N}$-atoms, and the aromatic ring of the target drug molecules yield hydrogen bonding, coordination interaction, and $\pi-\pi$ interaction with the unmodified Au NPs which mediates the aggregation. These

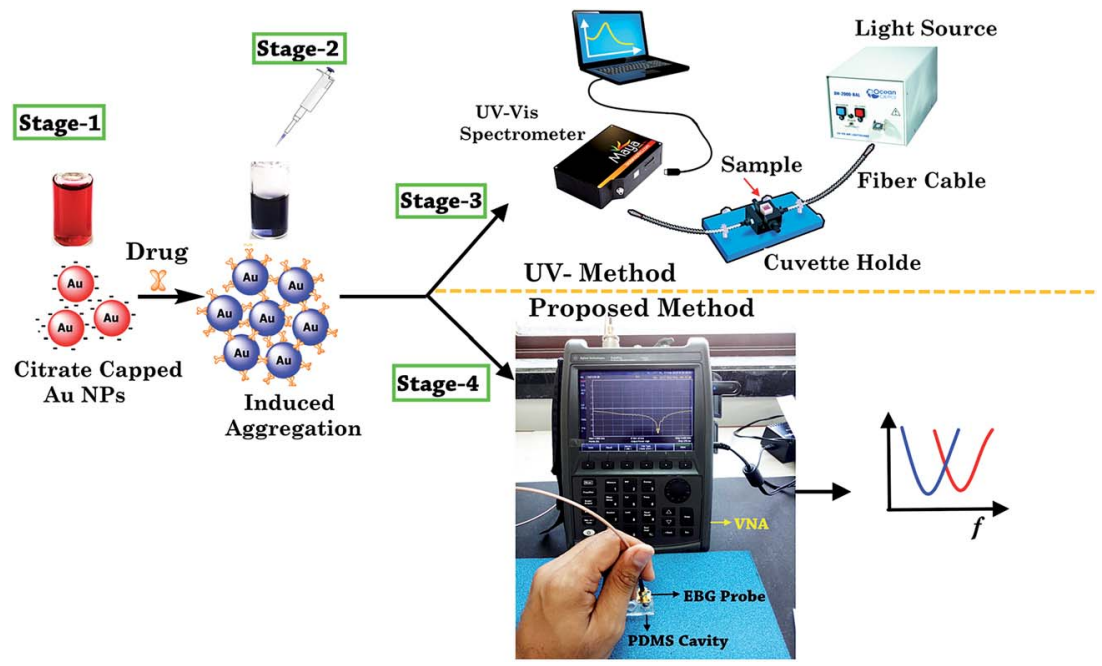

Fig. 6 Schematic of the sensing and measurement technique for the spectroscopy of two vasodilator drugs. 
conditions display higher sensitivity and selectivity for the detection of two vasodilator drugs. The effect of different buffer media on unmodified Au NPs was investigated to ensure that SPR phenomenon can take place solely due to the nanoparticles size, interparticle distance, the complex permittivity of the suitable buffer media, and the functional groups of the analyte drug. ESI Fig. $1 \dagger$ shows the absorption $\left(A_{\mathrm{SPR}} / A_{519}\right)$ of $\mathrm{Au}$ NPs in the presence of TRIS, ammonium acetate, and PBS buffer. It was found that PBS buffer changes the solution color to purple and peak absorption was measured at a longer wavelength $\left(\lambda_{825}\right)$. On the other hand, TRIS and ammonium acetate showed wavelength at $\lambda_{522}$ with an absorbance of 1.673 and 1.628. This shows that TRIS buffer maintains close similarity with the color and SPR band of the bare Au NPs and it can be used for the purpose of sensing.

UV-visible spectroscopy has been widely reported for the characterization of SPR phenomenon. It takes place in the nanoparticles due to the morphological changes. First of all, citrate as a reducing and stabilizing agent was used in the preparation of Au NPs with the sole objective of developing an alternate method for simple and sensitive detection of the analyte drugs. Further, drug solution was prepared with the help of aforesaid method, and the determined volume was injected into the Au NPs colloidal solution to observe the color change. The sample was characterized with a UV-visible method to confirm the complexing of Au NPs induced by the drug. After that, microwave spectroscopy was performed by transferring $200 \mu \mathrm{L}$ of the sample into a multi-compartment cavity made from polydimethylsiloxane (PDMS) and measuring the frequency of resonance on VNA by dipping the bandgapinspired probe into the sample. Fig. 6 shows the complete stages of the proposed method of analysis.

\subsection{Characterization}

Au NPs of 7-13 nm facilitate better binding ability of unmodified nanoparticles with the drug molecule. ${ }^{18}$ As shown in Fig. 7a, the average hydrodynamic diameter of the synthesized gold nanoparticles measured using DLS was found to be $\sim 11.5 \mathrm{~nm}$. A considerable increase in the average hydrodynamic diameter of the unmodified Au NPs was observed after modification with amlodipine and minoxidil. Fig. $7 \mathrm{~b}$ and $\mathrm{c}$ shows the increase in average diameter to $\sim 342$ and $\sim 105$ for amlodipine and minoxidil respectively. It indicates a decrease in the interparticle distance of Au NPs upon the addition of two drugs. This also indicates the presence of more binding site in the amlodipine drug compared to the minoxidil drug. The FT-IR spectroscopy was also studied to validate the organic framework on the surface of Au NPs and sensing mechanism.

ESI Fig. $2 \dagger$ shows the FT-IR spectrum of pure amlodipine, pure minoxidil, and their complexing with unmodified Au NPs at $\mathrm{pH}=4$ buffer media. The FT-IR spectra of amlodipine besylate and minoxidil were recorded in the solid-state form, whereas, the $\mathrm{Au}$ NPs sample was first mixed with potassium bromide, and then dried in the oven for $1 \mathrm{~h}$ for recording the spectra in a solid-state form. It is revealed from ESI Fig. $2 \mathrm{a}, \dagger$ that the peak at $3155 \mathrm{~cm}^{-1}$ corresponds to the $\mathrm{N}-\mathrm{H}$ stretching of
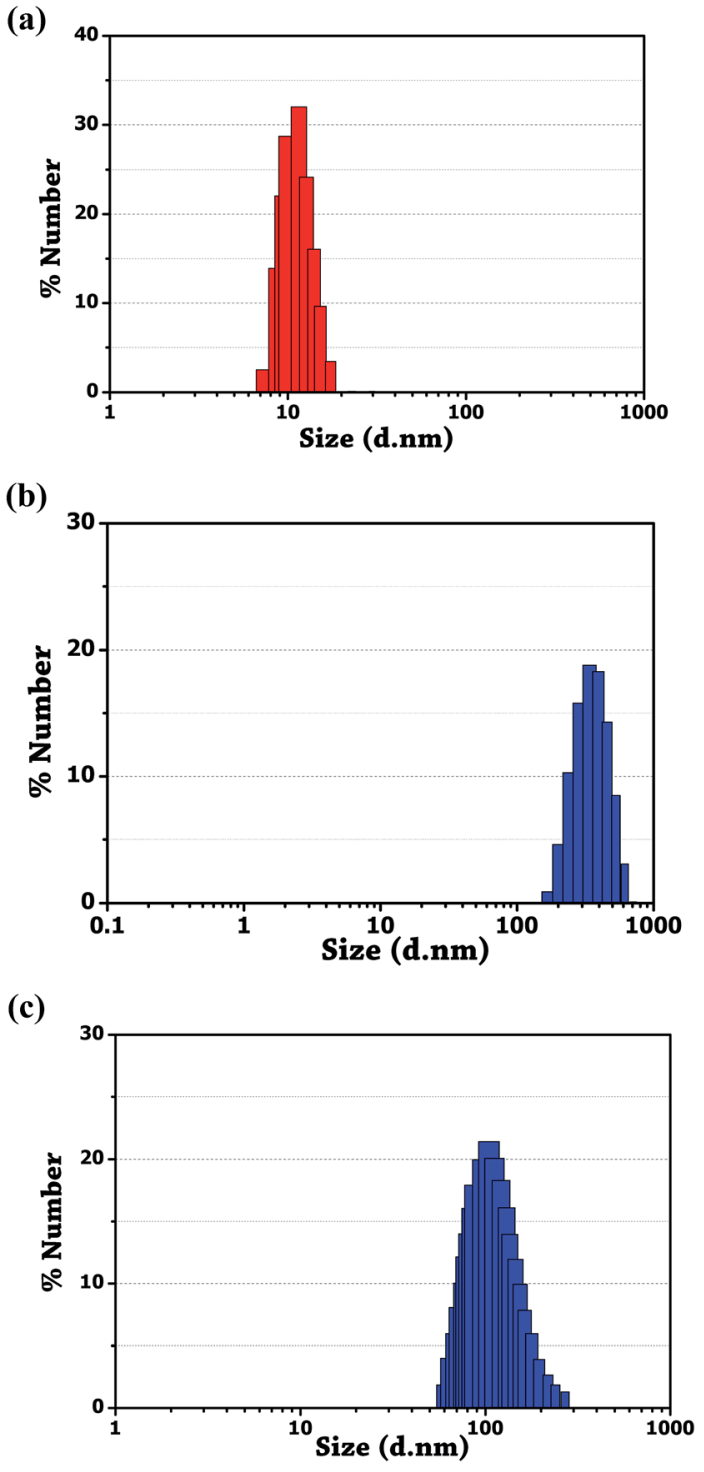

Fig. 7 DLS measurement. (a) Bare Au NPs. (b) Aggregation of unmodified Au NPs induced by amlodipine besylate. (c) Minoxidil.

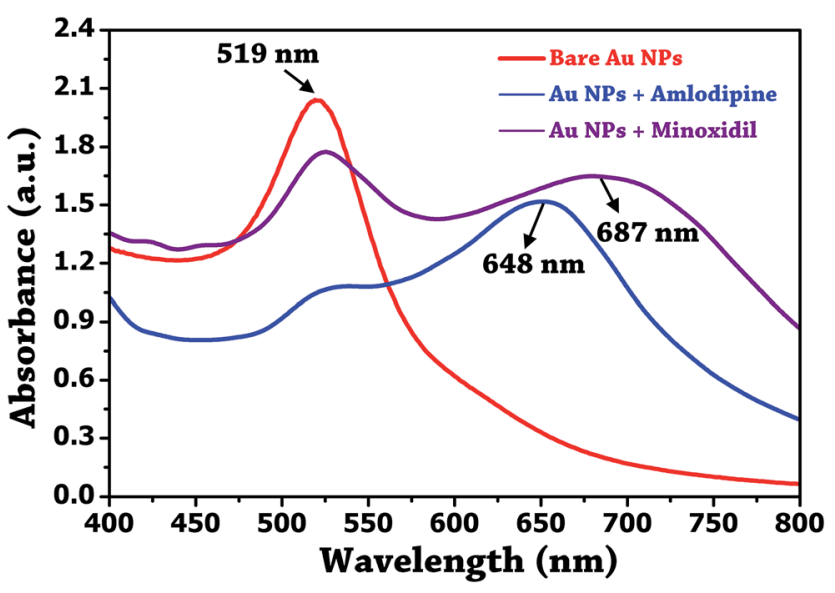

Fig. 8 UV-visible absorption plot of unmodified Au NPs, and their conjugation with amlodipine besylate $(100 \mu \mathrm{M})$ and minoxidil (10 mM). 
(a)

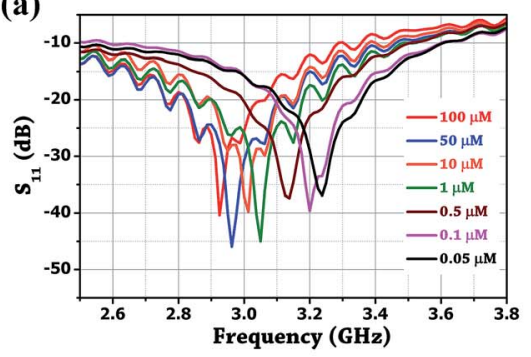

(b)

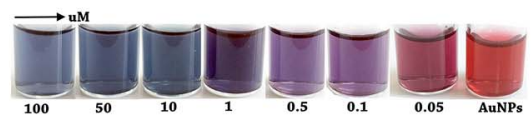

(c)

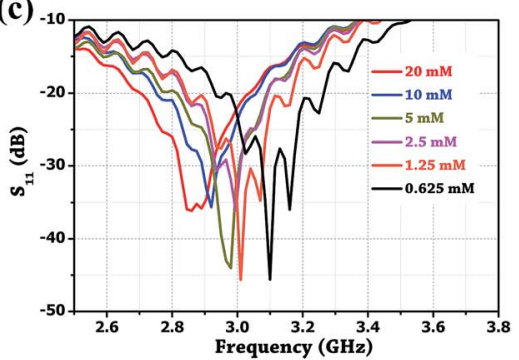

(d)

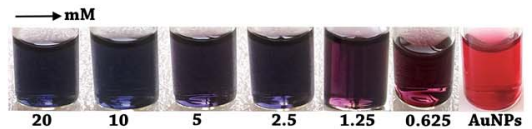

(e)

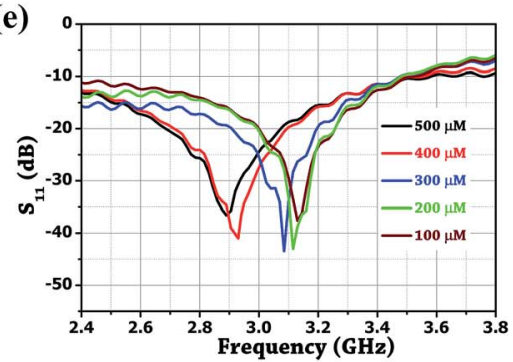

(f)

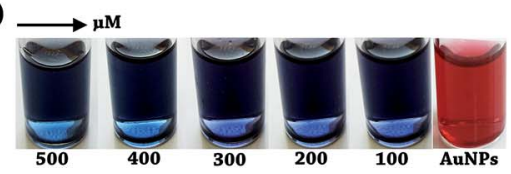

Fig. 9 Microwave resonance spectra and inset photographs of the corresponding Au NPs solution upon addition of (a and b) amlodipine besylate $(0.05-100 \mu \mathrm{M})$, (c and d) minoxidil $(0.625-20 \mathrm{mM})$, (e and f) amlodipine besylate $(100 \mu \mathrm{M})$ in the presence of minoxidil (100-500 $\mu \mathrm{M})$.

a primary amine group, and the peaks at $2948 \mathrm{~cm}^{-1}$ and 1614 $\mathrm{cm}^{-1}$ resembles the aromatic $\mathrm{C}-\mathrm{H}$ and $-\mathrm{C}=\mathrm{C}-$ stretching vibrations for the pure amlodipine besylate pharmaceutical drug formulation. Similarly, the peaks at $3423.72 \mathrm{~cm}^{-1}$ and $1643.98 \mathrm{~cm}^{-1}$ represents the $\mathrm{N}-\mathrm{H}$ stretching and bending of primary amines respectively in the pure minoxidil drug. More importantly, a drastic decrease in the intensity of theses peaks was observed in the spectra of Au NPs complex with amlodipine and minoxidil as shown in ESI Fig. $2 \mathrm{~b}$ and $d . \dagger$

The above results confirm the involvement of active function group $-\mathrm{NH}_{2}$, and benzene ring of the amlodipine in the induced aggregation and shift in the SPR band. The UV-visible spectrum was also recorded to ascertain the aggregation mechanism as shown in Fig. 8. The shape of the absorption profile of Au NPs was complete lost upon the addition of $100 \mu \mathrm{M}$ amlodipine besylate and $10 \mathrm{mM}$ minoxidil.

4.3 Analysis and detection of vasodilator drug formulations: microwave spectroscopy of Au NPs colorimetric probe using resonant probe

Initially, the colorimetric assay on the two drugs (amlodipine besylate and minoxidil) of different concentrations was performed individually using unmodified gold nanoparticles. It was done with the purpose of studying the extent especially when the colour change of the solution becomes difficult to analyze with naked eyes. In brief, a stock solution of amlodipine besylate $(100 \mu \mathrm{M})$ and minoxidil $(20 \mathrm{mM})$ was prepared using $\mathrm{pH} 4$ TRIS buffer. We believe that for the condition $\mathrm{p} K_{\mathrm{a}}$ citrate $<$ $\mathrm{pH} 4<\mathrm{p} K_{\mathrm{a}}$ 8.6, a strong electrostatic force of attraction exists between the Au NPs and two vasodilator drugs in concern. Therefore, various concentrations of amlodipine besylate were prepared from $100 \mu \mathrm{M}$ downwards, and the corresponding color changes were recorded. Fig. 9b shows the color change of solution from blue to purple and then magenta upon adding $100 \mu \mathrm{L}$ of the drug into $500 \mu \mathrm{L}$ of Au NPs in the range of 100$0.05 \mu \mathrm{M}$. Immediately, microwave spectroscopy was performed by measuring the reflection coefficient $\left(S_{11}\right)$ of each sample using the proposed metallic crystal loaded microwave resonant probe. As shown in Fig. 9a, the resonant frequencies were obtained at $2.925 \mathrm{GHz}, 2.9625 \mathrm{GHz}, 3.0125 \mathrm{GHz}, 3.05 \mathrm{GHz}$, $3.1735 \mathrm{GHz}, 3.2 \mathrm{GHz}$ and $3.2375 \mathrm{GHz}$ for the amlodipine besylate concentration in the range from 100-0.05 $\mu \mathrm{M}$. The spectroscopic observations were found consistent with the color change in Au NPs solution from red through magenta, purple and then to blue. As a result, the fitted linear equation $y=$ $0.001 x+0.382$ in the range of $100-10 \mu \mathrm{M}$ has $R^{2}=0.9787$ and
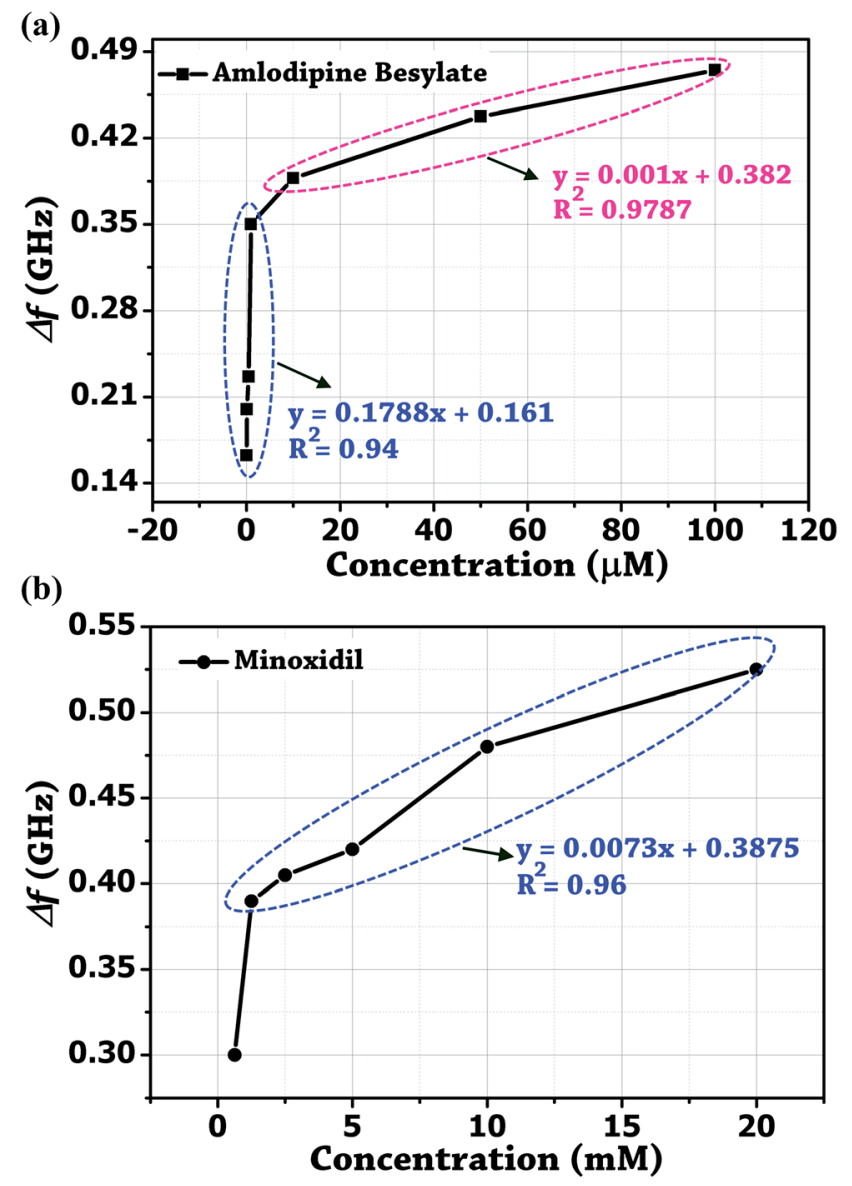

Fig. 10 Derived calibrated curves from the probe resonance for different concentration of the two vasodilator drugs added independently into Au NPs solution. (a) Amlodipine Besylate. (b) Minoxidil. 
Table 1 Analytical data obtained for the two vasodilator drugs using unmodified Au NPs as colorimetric probe and MPC-inspired sensor probe

\begin{tabular}{llll}
\hline & $\begin{array}{l}\text { Unmodified } \\
\text { Au NPs }\end{array}$ & $\begin{array}{l}\text { Au NPs aggregation } \\
\text { by amlodipine } \\
\text { besylate }\end{array}$ & $\begin{array}{l}\text { Au NPs } \\
\text { aggregation by } \\
\text { minoxidil }\end{array}$ \\
\hline $\begin{array}{llll}\text { Color } \\
\text { Size }(\mathrm{nm})\end{array}$ & Red & Blue & Blue \\
SPR (nm) & 11.57 & $\sim 342$ & $\sim 105$ \\
$\begin{array}{l}\text { Probe resonant } \\
\text { frequency (GHz) }\end{array}$ & 519 & 648 & 687 \\
$\begin{array}{l}\text { Active functional } \\
\text { group in }\end{array}$ & - & 2.925 & 2.875 \\
$\begin{array}{l}\text { aggregation } \\
\text { mechanism }\end{array}$ & & $-\mathrm{NH}_{2}$ & $-\mathrm{NH}_{2}$ \\
LOD & & & \\
\hline
\end{tabular}

Table 2 Repeatability and reproducibility analysis of the vasodilator drugs $^{a}$

\begin{tabular}{llll}
\hline Sample & $\begin{array}{l}\text { Concentration } \\
(\mu \mathrm{M})\end{array}$ & $\begin{array}{l}\text { Resonant frequency } \\
(\mathrm{GHz})\end{array}$ & $\begin{array}{l}\text { Precision }^{c} \\
(\%)\end{array}$ \\
\hline Drug-1 & 10 & $3.0125 \pm 0.03$ & 0.995 \\
& 0.5 & $3.1735 \pm 0.025$ & 0.787 \\
Drug-2 & 625 & $3.1 \pm 0.041$ & 1.322 \\
& 100 & $3.25 \pm 0.015$ & 0.461
\end{tabular}

${ }^{a}$ Drug-1: amlodipine besylate, drug-2: minoxidil. ${ }^{b}$ Mean \pm standard deviation $(n=3) .{ }^{c}$ Precision determined through \% RDS as (standard deviation $\div$ mean $) \times 100$.

linear equation $y=0.1788 x+0.161$ shows $R^{2}=0.94$ for concentration in the range $10-0.05 \mu \mathrm{M}$ (Fig. 10a). The calculated LOD was found to be $164 \mathrm{nM}$. This shows that as the drug concentration decrease, the solution permittivity also reduces which shifts the frequency of resonance towards the higher side in the frequency spectrum and near to the frequency response of bare Au NPs measured at 3.4 GHz.

Similarly, the conjugation of Au NPs with minoxidil drug was investigated for different concentrations between $20-0.625 \mathrm{mM}$. Fig. 9d shows a progressive change in the Au NPs solution color from red to blue. Testing at the further lower concentration was not favorable due to low solubility of the drug which may otherwise result in erroneous reading. Through the in situ analysis using the proposed microwave resonant probe, the frequency of resonances were obtained at $2.875 \mathrm{GHz}, 2.92 \mathrm{GHz}$, $2.98 \mathrm{GHz}, 2.995 \mathrm{GHz}, 3.01 \mathrm{GHz}$ and $3.1 \mathrm{GHz}$ in the range of 20$0.625 \mathrm{mM}$ drug respectively as shown in Fig. 9c. The linear trend between the resonant frequencies $\left(f_{\mathrm{r}}\right)$ and drug concentration is found to be $y=0.0073 x+0.3875$, resulting in the coefficient of determination $R^{2}=0.96$ with estimated LOD $=1.78 \mathrm{mM}$ (Fig. 10b). The response of the probe was also tested for the coexistence of minoxidil $(100-500 \mu \mathrm{M})$ in amlodipine $(100 \mu \mathrm{M})$ in the solution ratio $1: 2$. It was observed that the presence of minoxidil did not influence the binding of amlodipine with $\mathrm{Au}$ NPs, and distinct resonances were obtained, viz. $2.89 \mathrm{GHz}, 2.93$
$\mathrm{GHz}, 3.085 \mathrm{GHz}, 3.115 \mathrm{GHz}$, and $3.13 \mathrm{GHz}$ as shown in Fig. 9e and f. Table 1 summarizes the analytical data obtained from the analysis of two drugs.

The performance of the probe was ascertained by repeating the experiment with three iterations $(n=3)$ on different samples. The measured resonances for amlodipine besylate (10 $\mu \mathrm{M}, 0.5 \mu \mathrm{M})$, and minoxidil $(625 \mu \mathrm{M}, 100 \mu \mathrm{M})$ have lower standard deviation as shown in Table 2 . The precision determined through relative standard deviation (RSD) was found $<2 \%$ and demonstrates less uncertainty between the measurements. In the results of probe reflection coefficient $\left(S_{11}\right)$ for the two vasodilator drugs, ripples in the resonance curve are likely due to the continuous aggregation of $\mathrm{Au}$ NPs over time. However, one should note that the resonant frequency points will be unaffected, as the probe sensor is inspired by the electromagnetic bandgap properties which restrict any deviation in the frequency of resonance. But, a lower value of standard deviation still exists, which might be due to the slight error in the position of the probe, and can be corrected using a position control system or a fixed clamp-type arrangement. Our results suggest that the proposed analytical method of microwave spectroscopy in combination with the colorimetric assay of gold nanoparticles can be effectively used for the simple detection and quantification of the analyte drugs.

\section{Conclusions}

A very economical, time-effective and simple analytical technique based on microwave spectroscopy of unmodified Au NPs undergoing aggregation upon the addition of analyte at optimum $\mathrm{pH}$, has been reported in the present work. Our proposed technique involving the distinct resonance curves for different concentration of the two drugs in Au NPs, is much simpler and user-friendly than that of UV-visible spectroscopy. We demonstrated the detection and quantification of two vasodilator drugs (amlodipine besylate and minoxidil) in their pharmaceutical formulations, using unmodified $\mathrm{Au}$ NPs. The applicability of our proposed technique can be further enhanced to improve LOD (currently $164 \mathrm{nM}$ and $1.78 \mathrm{mM}$ for amlodipine besylate and minoxidil, respectively) of the targeted drugs by using surface-modified Au NPs, janus nanoparticles, functionalized nanoparticles, etc. which may provide larger scope of future work. Ripple in the resonance curves is one of the identified challenge which occurs due to the dispersive nature of Au NPs solution. The method was successfully tested in the case where two drugs may coexist. The repeatability and reproducibility test shows higher amount of precision has been achieved. It anticipated that the analytical platform with integrity of microwave sensor probe and Au NPs colorimetric probe simultaneously holds potential for the analysis of drugs, biomolecules, and pathogens.

\section{Acknowledgements}

The authors thank Electronics Engineering Department and Chemical Engineering Department, SVNIT, for providing the measurement facility. They also thank Technical Quality 
Improvement Program (TEQIP), Phase-II, SVNIT, for supporting this research (Grant no. ECED/TEQIP-II/1910/2015-2016).

\section{References}

1 M. Miyamoto, K. Kotani, S. Ishibashi and N. Taniguchi, Int. J. Vasc. Med., 2012, 2012, 1-11.

2 J. N. Cohn MD, G. T. Mclnnes MD and A. M. Shepherd MD, J. Clin. Hypertens., 2011, 13, 690-692.

3 S. Julius, S. E. Kjeldsen and M. Weber, Lancet, 2004, 363, 2022-2031.

4 L. Amankwa, L. G. Chatten and S. Pons, Analyst, 1983, 108, 1221-1226.

5 R. A. Shaalan, T. S. Belal, F. A. El Yazbi and S. M. Elonsy, Arabian J. Chem., 2017, 10, 381-394.

6 L. Gagliardi, A. Amato, L. Turchetto and D. Tonelli, Anal. Lett., 1991, 24, 1825-1835.

7 R. R. Kallem, R. Mullangi, K. K. Hotha, L. K. Ravindranath, Y. N. Spoorthy and J. V. L. N. Seshagirirao, Bioanalysis, 2013, 5, 827-837.

8 D. R. Johnson and S. R. Botch, J. Anal. Toxicol., 2011, 35, 6574.

9 N. Y. Khalil, T. A. Wani, M. A. Abunassif and I. A. Darwish, J. Liq. Chromatogr. Relat. Technol., 2011, 34, 2583-2595.

10 I. A. Aljuffali, C. T. Sung, F.-M. Shen, C.-T. Huang and J.-Y. Fang, AAPS J., 2014, 16, 140-150.

11 M. Khairy, A. A. Khorshed, F. A. Rashwan, G. A. Salah, H. M. A. Abdel-Wadood and C. E. Banks, Sens. Actuators, B, 2017, 239, 768-775.

12 V. Pfaffeen and P. I. Ortiz, Anal. Sci., 2005, 22, 91-94.

13 N. Alizadeh and F. Hemati, Bull. Fac. Pharm., 2014, 52, 109114.

14 Z. A. Zaheer, S. Mirza, I. Moazzam and I. W. Sayad, Der Pharma Chemica, 2012, 4, 568-573.

15 K. Saha, S. S. Agasti, C. Kim, X. Li and V. M. Rotello, Chem. Rev., 2012, 112, 2739-2779.

16 Y.-C. Yeh, B. Creran and V. M. Rotello, Nanoscale, 2012, 4, 1871-1880.

17 K. A. Rawat, H. Basu, R. K. Singhal and S. K. Kailasa, RSC Adv., 2015, 5, 19924-19932.

18 S. Teepoo, P. Chumsaeng, K. Palasak, N. Bousod, N. Mhadbamrung and P. S. Lim, Talanta, 2013, 117, 518-522.
19 M. Yarbakht and M. Nikkhah, J. Exp. Nanosci., 2016, 11, 593601.

20 X. Xie, R. Deng, F. Liu, W. Xu, S. F. Y. Li and X. Liu, Anal. Methods, 2013, 5, 1116-1119.

21 V. Selvaraj, M. Alagar and I. Hamerton, Electrochim. Acta, 2006, 52, 1152-1160.

22 K. Grenier, D. Dubuc, P.-E. Poleni, M. Kumemura, H. Toshiyoshi, T. Fujii and H. Fujita, IEEE Trans. Microwave Theory Tech., 2009, 57, 3246-3253.

23 N.-Y. Kim, K. K. Adhikari, R. Dhakal, Z. Chuluunbaatar, C. Wang and E.-S. Kim, Sci. Rep., 2014, 5, 1-9.

24 Y.-F. Chen, H.-W. Wu, Y.-H. Hong and H.-Y. Lee, Biosens. Bioelectron., 2014, 61, 417-421.

25 D. Smith, W. Padilla, D. Vier, S. N. Nasser and S. Schultz, Phys. Rev. Lett., 2000, 84, 4184-4187.

26 C. Cao, J. Zhang, X. Wen, S. L. Dodson, N. T. Dao, L. Mun Wong, S. Wang, S. Li, A. T. Phan and Q. Xiong, Nano, 2013, 7, 7583-7591.

27 S. J. Park, J. T. Hong, S. J. Choi, H. S. Kim, W. K. Park, S. T. Han, J. Y. Park, S. Lee, D. S. Kim and Y. H. Ahn, Sci. Rep., 2014, 4, 1-7.

28 H.-J. Lee, J.-H. Lee and H.-L. Jung, Appl. Phys. Lett., 2011, 99, 163703.

29 J. P. Grant, R. N. Clarke, G. T. Symm and N. M. Spyrou, J. Phys. E: Sci. Instrum., 1989, 22, 757-770.

30 M. Stuchly, T. Athey, G. Samaras and G. Taylor, IEEE Trans. Microwave Theory Tech., 1982, 30, 87-92.

31 D. Baudry, A. Louis and B. Mazari, Prog. Electromagn. Res., 2006, 60, 311-333.

32 D. J. Rowe, A. Porch, D. A. Barrow and C. J. Allender, IEEE Trans. Microwave Theory Tech., 2012, 60, 1699-1708.

33 N. Wagner, M. Schwing and A. Scheuermann, IEEE Trans. Geosci. Rem. Sens., 2014, 52, 880-893.

34 A. R. McGurn and A. A. Maradudin, Phys. Rev., 1993, 48, 17576-17579.

35 E. I. Smirnova, C. Chen, M. A. Shapiro, J. R. Sirigiri and R. J. Temkin, J. Appl. Phys., 2002, 91, 960-968.

36 J. Turkevich, P. C. Stevenson and J. Hillier, Discuss. Faraday Soc., 1951, 11, 55-75.

37 https://www.drugbank.ca/drugs/DB00381.

38 https://www.drugbank.ca/drugs/DB00350. 